University of Wollongong

Research Online

Australian Institute for Innovative Materials -

Papers

Australian Institute for Innovative Materials

$1-1-2016$

\title{
3D printed flexure hinges for soft monolithic prosthetic fingers
}

Rahim Mutlu

University of Wollongong, rmutlu@uow.edu.au

Gursel Alici

University of Wollongong, gursel@uow.edu.au

Marc in het Panhuis

University of Wollongong, panhuis@uow.edu.au

Geoffrey M. Spinks

University of Wollongong, gspinks@uow.edu.au

Follow this and additional works at: https://ro.uow.edu.au/aiimpapers

Part of the Engineering Commons, and the Physical Sciences and Mathematics Commons

Research Online is the open access institutional repository for the University of Wollongong. For further information contact the UOW Library: research-pubs@uow.edu.au 


\title{
3D printed flexure hinges for soft monolithic prosthetic fingers
}

\begin{abstract}
Mechanical compliance is one of the primary properties of structures in nature playing a key role in their efficiency. This study investigates a number of commonly used flexure hinges to determine a flexure hinge morphology, which generates large displacements under a lowest possible force input. The aim of this is to design a soft and monolithic robotic finger. Fused deposition modeling, a low-cost 3D printing technique, was used to fabricate the flexure hinges and the soft monolithic robotic fingers. Experimental and finite element analyses suggest that a nonsymmetric elliptical flexure hinge is the most suitable type for use in the soft monolithic robotic finger. Having estimated the effective elastic modulus, flexion of the soft monolithic robotic fingers was simulated and this showed a good correlation with the actual experimental results. The soft monolithic robotic fingers can be employed to handle objects with unknown shapes and are also potential low-cost candidates for establishing soft and one-piece prosthetic hands with light weight. A three-finger gripper has been constructed using the identified flexure hinge to handle objects with irregular shapes such as agricultural products.
\end{abstract}

\section{Keywords}

3d, printed, flexure, prosthetic, hinges, fingers, soft, monolithic

\section{Disciplines}

Engineering | Physical Sciences and Mathematics

\section{Publication Details}

Mutlu, R., Alici, G., in het Panhuis, P. \& Spinks, G. M. (2016). 3D printed flexure hinges for soft monolithic prosthetic fingers. Soft Robotics, 3 (3), 120-133. 


\title{
3D Printed Flexure Hinges for Soft Monolithic Prosthetic Fingers
}

\author{
Rahim Mutlu, Gursel Alici, Marc in het Panhuis, and Geoffrey M. Spinks
}

\begin{abstract}
R. Mutlu (rmutlu@uow.edu.au), G. Alici ("Corresponding Author, gursel@uow.edu.au.), and G. Spinks (gspinks@uow.edu.au) are with School of Mechanical, Materials and Mechatronic Engineering, and Intelligent Polymer Research Institute, ARC Centre of Excellence for Electromaterials Science, University of Wollongong, AIIM Facility, NSW, 2522, Australia.

M. in het Panhuis (panhuis@uow.edu.au) is with the Soft Materials Group, School of Chemistry and Intelligent Polymer Research Institute, ARC Centre of Excellence for Electromaterials Science, University of Wollongong, AIIM Facility, NSW, 2522, Australia.
\end{abstract}

\begin{abstract}
Mechanical compliance is one of the primary properties of structures in nature playing a key role in their efficiency. This study investigates a number of commonly used flexure hinges in order to determine flexure hinge morphology which generates larger displacements under the lowest force input. The aim of this is to design a soft and monolithic robotic finger. Fused deposition modelling (FDM), a low cost 3D printing technique, was used in order to fabricate the flexure hinges and the soft monolithic robotic fingers. Experimental and finite element analyses suggest that a non-symmetric elliptical flexure hinge is the most suitable type for use in the soft monolithic robotic finger. Having estimated the effective elastic modulus, flexion of the soft monolithic robotic fingers was simulated and this showed a good correlation with the actual experimental results. The soft monolithic robotic fingers can be employed to handle objects with unknown shapes and are also potential low cost candidates for establishing soft and one-piece prosthetic hands with light weight. A 3-finger gripper has been constructed using the identified flexure hinge to handle objects with irregular shapes such as agricultural products.
\end{abstract}




\section{Introduction}

Biological species such as animals and some plants convert metabolic energy into mechanical work using their soft and/or compliant limbs which have integrated in them a mechanical structure, actuation, sensing and control features. These features are all strategically combined as a single functioning unit which generates smooth actions and adapts well to various terrain conditions and environments. This is the desired behavior of a robotic device with these features. On the other hand, engineered mechanisms and machines have been established by employing very stiff or completely rigid components connected to each other via joints in order to convert the input energy into a mechanical output. Mimicking animals' movements, the body of a robot can be fabricated as a one piece compliant mechanism which can convert the input energy in the form of a mechanical displacement and force into an output motion via its relatively flexible bending points. The flexible members of a compliant mechanism are designed to be slender so that, for a given mechanical input, a relatively large deformation occurs at those flexible members which are called flexure hinges or joints [1]. Deformation at other regions of the compliant mechanism is negligible as these regions remain relatively rigid under a given mechanical input. Compliant mechanisms can be divided into two major classes: (i) semi-compliant mechanisms and (ii) fully compliant mechanisms. In a semicompliant mechanism, flexible or slender members can be fabricated from different materials (usually flexible materials such as stainless steel, titanium, flexible plastics) which have lower stiffness than the remaining parts of the compliant mechanism. In contrast, a fully compliant mechanism is fabricated from a single material as a one-piece structure. While a semi-compliant mechanism requires additional fabrication tasks such as assembly of flexible members, a fully compliant mechanism is more cost effective because no additional tasks are required to complete the compliant mechanism. Even though compliant mechanisms, either semi-compliant or fully compliant, have found numerous engineering applications, a fully compliant mechanism is commonly preferred in milli- and micro-domain applications where the assembly of a mechanism is topologically sophisticated [2-6]. Sreetharan et al. demonstrated a MEMS fabrication technique incorporated with origami folding approach to fabricate and assemble a monolithic flapping wing robotic insect [7]. Compliant mechanisms are usually used in these applications because the deformation of a flexure hinge is generally small. In macro-domain robotic applications, hyperelastic materials can be used to fabricate a compliant robot with flexure hinges where larger deformations are achievable without a plastic deformation in the flexure hinges.

A human hand, as a biological system, is a highly dexterous device that helps us not only to manipulate an object but also to identify some of the properties of the object, and interact with our environment. There have been numerous technological developments in prostheses to provide this ability to those who have lost their limbs or part of their limbs. Although commercially available advanced prosthetic devices, particularly prosthetic hands, have been on the market for some time, they are still not close to a real human hand in terms of performance or dexterity. A human hand is not only a dexterous gripper with 14 of degrees of freedom (DoF), but it also has a neurological network which feels an object, and provides haptic feedback about the object being handled. Mimicking such a system in a prosthetic hand requires very complex nonlinear control to accurately 
manipulate and identify an object. The classical approach based on directly copying from the biological counterpart has limitations such as availability of smart and functional materials which exhibit similar properties as biological materials. We need innovative approaches for seamless fabrication with smart materials and structures mimicking that capture the behavior of the real biological system. Soft robotics, which focuses mostly on biologically inspired robotics using soft bodies, has been gaining significant interest in the field of robotics as well as in other scientific areas of research. For instance, while a soft robotic tentacle can adaptively grasp and manipulate an object with no damage to the object, a classic robotic manipulator must use many design and control algorithms in order to perform the same task safely but this property is necessary if the device will perform its tasks together with or in close proximity to a human being. Soft robotic devices also have the properties of programmable compliance and adaptability, properties which are particularly important for applications where the robotic device interacts directly with humans. The compliance in the structure of a soft robotic device or mechanism usually comes from the intrinsic properties of the soft material being used.

There have been a number of studies conducted in this area of robotics: on the motion of a snake [8-10], an octopus tentacle [11-13], an elephant trunk [14, 15] and an inch worm or caterpillar [1618], and similar motion generating species [19-21]. While there is a trend moving away from rigid robotic systems towards softer robotic systems, the soft robotic examples in the literature generally rely on external actuation systems such as electric motors with conventional rigid mechanisms as endoskeleton or tendons with a pulley system, or a pump. The soft robots which use an internal rigid mechanism or a pulley system with tendons driven by an electric motor have a sleeve made of a soft material (usually a silicone rubber) which gains softness from the external sleeve. The ones using a pump system generate their motion by pressurized fluid, filling the soft structure of the device through specially designed channels [19, 21-23]. Shape deposition manufacturing (SDM), as a rapid prototyping method, has been used in the literature to design and fabricate soft robotic fingers [24, 25]. Dollar and Howe designed an underactuated soft gripper with flexure hinges fabricated via SDM which is also based on tendon cable actuation routed in the fingers of the gripper [24]. Some examples of soft robotics are illustrated in Fig. 1.
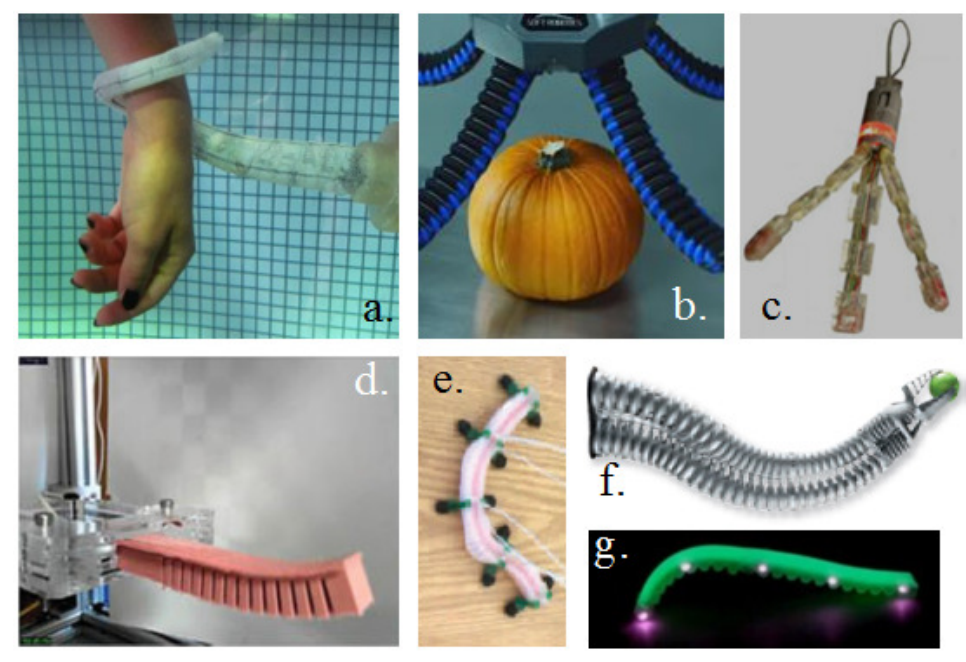
Fig. 1. Some examples of soft robotics: a. octopus arm [26], b. soft gripper [27], c. surgical grasper [25], d. pneumatic soft finger [21], e. snake [10], f. elepthant trunk [28] and caterpillar [18].

Soft robotic devices have been fabricated using traditional methodologies such as molding the soft parts of a robotic device where molds are fabricated using an additive manufacturing method $[19,21,22]$ or using an additive manufacturing method to directly fabricate some parts of the soft robotic device [28]. Additive manufacturing methods not only reduce the cost of fabrication but also enable us to fabricate highly complex geometries within the topology of a soft robotic device. It is more desirable to fabricate a biologically inspired soft robotic device from smart materials which offer built-in actuation and/or sensing in the same geometry or body. Mutlu et al. [29, 30] have recently reported on modelling a polypyrrole (PPy) based electroactive polymer actuator (EAP) as a one-piece active structure. We envision that having a structure or mechanism made of an electro-active material means or any smart and functional material that the entire mechanism can be fabricated from a single material providing both compliance and adaptability with no additional actuation required because of the built-in actuation property of the material while proving more biologically mimicked robotic devices. One disadvantage, however, of the actuators made of soft and smart materials such as ionic EAP actuators (aka artificial muscles) is that while they may be suitable for milli- and micro-domain robotic applications, they generate a relatively low force output and therefore, they might not be suitable for macro-domain applications. On the other hand, there are other types of artificial muscles such as dielectric elastomer actuators which generate a relatively large force output. A major drawback of these artificial muscles, however, is that they require a high voltage input (typically over $1 \mathrm{kV}$ ). Using additive manufacturing technology, soft smart materials can be formed into functional devices which will produce useful mechanical work in response to a given stimulus depending on the smart material, for example, using electric, magnetic field, temperature or light as the stimulus. The concept of 4D printing is based on printing a 3D structure from a smart material or materials so that the structure changes its geometry or configuration over time under a designated stimulus [31,32]. 4D printing is still in its infancy with a limited number of smart materials to be used for printing. The response time of the smart materials for 4D printing is also quite long. Until 3D printable soft smart materials with a short response time are developed, soft robotic device developers are restricted to using soft passive materials.

In this study, we use a 3D printable soft material (i.e. a thermoplastic elastomer) to establish an underactuated soft robotic finger which is fully compliant and adaptive, and can be used as the finger of a soft robotic gripper or a prosthetic hand. The main purpose of employing 3D printing fabrication method is that all number of fabrication steps are eliminated and desired form of robotic structure can be fabricated monolithically. We employed a commercially available low cost 3D printer (UP Plus 2), which is based on fused deposition modeling (FDM) additive manufacturing method, to fabricate the finger. Before fabricating the soft monolithic robotic finger, we investigated the bending behavior of various flexure hinges for the soft robotic finger in order to quantify the effect of the hinge design on its flexion-extension. The main objectives of having a monolithic 
robotic or prosthetic finger are to reduce the complexity of fabrication by eliminating unnecessary assembly steps, scalability and also to have an adaptive structure to improve conformability of the finger. A fully compliant robotic finger design will also reduce the total weight of the prosthetic hand because it is fabricated as a monolithic structure using the 3D printing which means that the porosity of the fingers and the hand is directly related to the overall weight of the hand. Weight and operating noise are two of the major problems which have an influence on the ease of use and hence the acceptability of prosthetic hands [33]. Developing prosthetic devices made of smart materials fabricated using additive manufacturing methods can enable us to overcome these difficulties. The primary contributions of this study are to quantify and model the bending behavior of flexural hinges and subsequently identify the flexural hinge which will produce the highest elastic potential energy under the same mechanical input. A monolithic soft robotic finger based on the identified flexure hinge is also designed and fabricated. The flexion and extension behaviors of the soft finger have also been studied. The modeling, design and fabrication methodology proposed in this study is offered as a guide for establishing functional soft robotic devices.

\section{Additive Manufacturing}

Additive manufacturing is a paradigm shift from subtractive manufacturing techniques such as molding, machining, forging to the advanced manufacturing techniques that a final product is produced by adding a material layer-by-layer according to the digital model of a real object. As a class of additive manufacturing methods, FDM has shown exponential growth in the last decade. FDM generally uses thermoplastic materials extruded layer-by-layer to form a 3D shape. The 3D printing platform and a soft monolithic robotic finger fabricated using the FDM method are shown in Fig. 2.

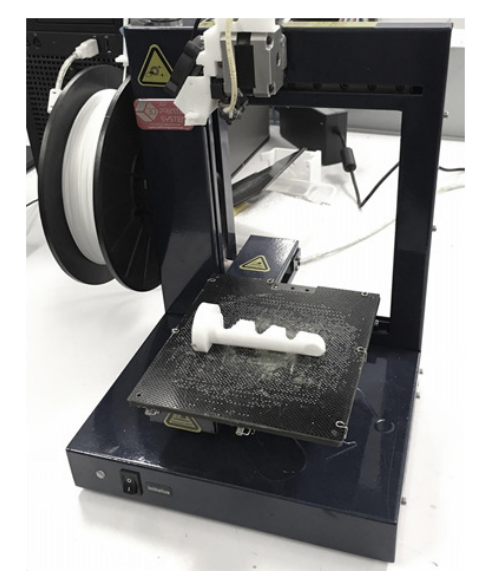

Fig. 2. A typical monolitic soft finger fabricated using FDM type 3D printer (3D Printing Systems, UP Plus 2) using TPU.

\section{Modelling the Flexure Hinge of the Soft Monolithic Finger}

Modelling the flexure hinges of the soft finger is important in order to estimate the mechanical behavior and to optimize its dimensions in order to generate the desired input-output relation of the 
whole finger. The flexure hinge, which is the most flexible part of the mechanism, undergoes relatively large deformations compared to other parts of the mechanism such as the finger. Even though flexure hinges are categorized into three classes: single-axis, double-axis and multiple-axis, the single-axis flexure hinges are mostly used in compliant mechanisms [1]. A single-axis flexure hinge works in 2D while other classes of the flexure hinges generate their motion in 3D. Flexure hinges are also categorized as symmetric and non-symmetric in terms of their geometry. Symmetric flexure hinges show a geometrical symmetry based on their longitudinal axis (i.e. $y$-axis in Fig. 3), and non-symmetric flexure hinges have one flat side and one with a designated geometrical shape. The most commonly used single-axis hinges in compliant mechanisms are corner-filleted, circular, and elliptical, as shown in Fig. 3.

(a)

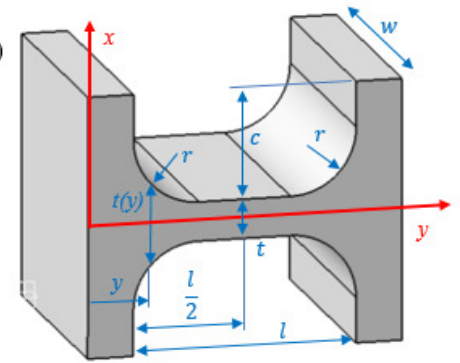

(c)

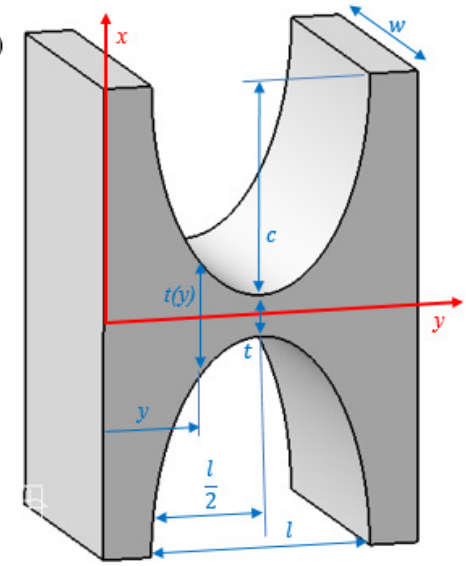

(b)

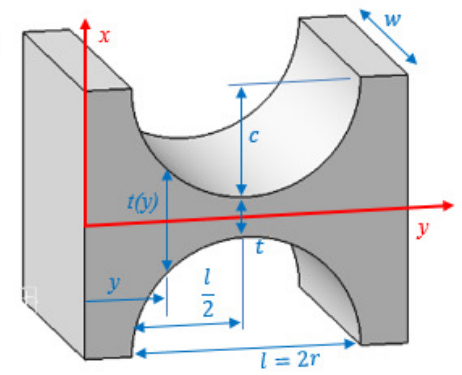

(d)

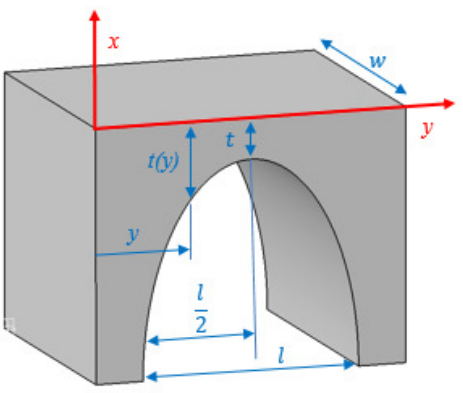

Fig. 3. Geometric parameters of single-axis flexure hinges: symmetric (a) corner-filleted, (b) circular, (c) elliptical and (d) non-symmetric elliptical.

\section{a. Geometric Description of Flexure Hinges}

Geometric relations for the thickness of the symmetric corner-filleted, circular, elliptical and non-symmetric flexure hinges are given as:

$$
t_{c f}(y)=\left\{\begin{array}{l}
t+2[r-\sqrt{y(2 r-y)}] \\
t \\
t+2\{r-\sqrt{(l-y)[2 r-(l-y)]}\}
\end{array}\right.
$$




$$
t_{c i r}(y)=t+2[r-\sqrt{y(2 r-y)}]
$$

and

$$
t_{e l}(y)=t+2 c\left[1-\sqrt{1-\left(1-\frac{2 y}{c}\right)^{2}}\right]
$$

The thickness of the corresponding non-symmetric single-axis flexure hinge can be calculated by halving the thickness of a symmetric single-axis flexure hinge defined by Equations 1-3 as one side of a non-symmetric flexure hinge is flat $[1,34]$ :

$$
t_{\text {non }}(y)=\frac{t(y)+t}{2}
$$

where $t$ is the thickness of the flexure hinge at its thinnest point, $\mathrm{c}$ is the depth of the hinge groove, $r$ is the fillet radius which is equal to $c$ for a circular flexure hinge, $l$ is the length of the hinge groove and $w$ is the width of the flexure hinge.

\section{b. Estimating the Effective Young's Modulus of FDM Materials}

We employ finite element modelling (FEM) to quantify and model the bending capabilities of the flexure hinges. FE models are usually used for continuum bodies or structures with homogenous materials where the material properties are consistent throughout the structure being modelled. Using the FDM type additive manufacturing method, parts are fabricated with porosity in order to reduce the overall weight of an object. We use FE models of the flexure hinges to first estimate the effective Young's modulus of the thermoplastic elastomer and subsequently simulate the mechanical behavior of the soft finger which is 3D printed using the same thermoplastic elastomer under the same printing conditions. FE models of the flexure hinges have been built by employing the geometric relations given by Equations 1-4 and their bending capabilities have been analyzed. In the FE simulations, the flexure hinge is fixed at one end and a pulling force is applied at the other end, in the mid-point of the width of the flexure hinge, as illustrated in Fig. 4.

Similar to the simulation configuration, a range of forces were experimentally applied to the flexure hinge and their bending displacements were obtained. The angular and Cartesian displacement results of the flexure hinge were obtained from the surface of the free end and from point A, respectively (Fig. 4), where pulling force was applied. Under the same force applied to each flexure hinge, the bending moment of the flexure hinge is calculated at the center of the flexure hinge and this allows us to make a comparison between different hinge designs in terms of the applied bending moment and the resulting displacement. The details of the input force, bending moment acting at the center point (' $O$ ') of the flexure hinge and bending displacement are illustrated in Fig. 4. The displacements are measured from point ' $A$ ', $\theta$ is the force angle, $\beta$ is the bending angle and $\emptyset$ is the angle between the input force and the force, $F_{M}$, acting perpendicular to the

moment arm, $|O A|$. The moment arm $|O A|$ is assumed to be constant during the bending of the 
flexure hinge whereas slight deformations might occur in other regions of the flexure hinge. The bending moment at center point $O$ is calculated using the moment arm $|O A|$.

We have used one of the flexure hinges to identify the Young's modulus of the thermoplastic used to fabricate the 3D printed flexure hinges. By comparing the displacement results from the FE model of the flexure hinge with the experimental results from the 3D printed flexure hinge, we have estimated the effective Young's modulus of the printed TPE. Two groups of flexure hinges which were 3D printed under different printing settings were used to compare their bending angle and displacement capabilities. The effective Young's moduli of test group 1 (G1) and test group 2 (G2) flexure hinges are estimated. A comparison of the experimental and numerical displacement results for the flexure hinges are presented in Section V.
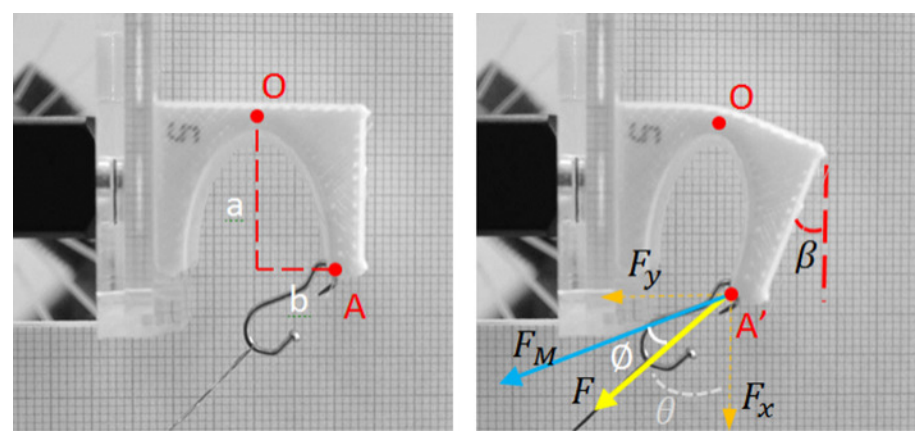

Fig. 4. Diagram for the input force - bending moment relation for a typical non-symmetric elliptical flexure hinge (hinge is at the initial state on the left and at a bent state on the right).

\section{c. Geometric Parameters for the Flexure Hinges and the Soft Fingers}

The geometric parameters of the flexure hinge and the soft robotic finger used are based on those of a human finger [35]. In this study, we have used an index finger as a reference for the size of the robotic finger. It should be noted that the geometric parameters of a human finger (thickness and width) vary along the length of the finger. The geometric parameters of a typical human index finger are given in Fig. 5 [35, 36].

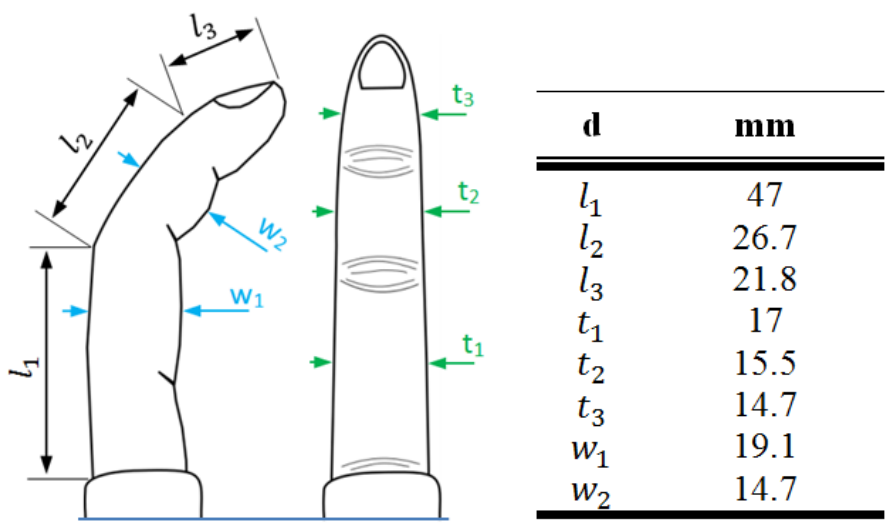

Fig. 5. Geometric parameters of a human index finger. 
The flexure hinges are designed to fit in the soft robotic finger as well as to provide enough compliance for the flexion and extension of the robotic finger. Thickness $t$ (the thinnest region of the flexure hinge) of the hinges is $3 \mathrm{~mm}$ which allows not only a compliant motion transfer through the flexure hinges but also provides stability so that buckling will not occur during actuation (especially flexion) of the soft finger.

\section{Designing a Soft Robotic Finger: 3D Printed with Thermoplastic Elastomer}

The non-symmetric elliptical type flexure hinge shows the largest bending angle and the highest bending displacement compared with other type flexure hinges under the same input force. The soft monolithic finger has been designed with three of these flexure hinges, resembling the human index finger, and it is actuated with one tendon cable passing through the designated channels within the finger. Such configuration enables us to design a simple finger structure to be fabricated as a onepiece compliant body and to build an adaptive robotic finger which can be underactuated (where the number of the actuators is lower than the number of DoF; the three hinges are activated through one tendon). The concept of underactuation is essential to reduce number of actuators needed to actuate a multi-finger gripper or a robotic hand, hence significantly reduce the total weight and size of the robotic or prosthetic hand. Rather than using one actuator per finger, one actuator can activate all fingers simultaneously - having underactuation fingers conform onto the surface of an object while grasping is performed. The distance between the channels for tendons and the thinnest region of the flexure hinge is designed as large as possible in order to increase the bending moment generated by the tendon force.

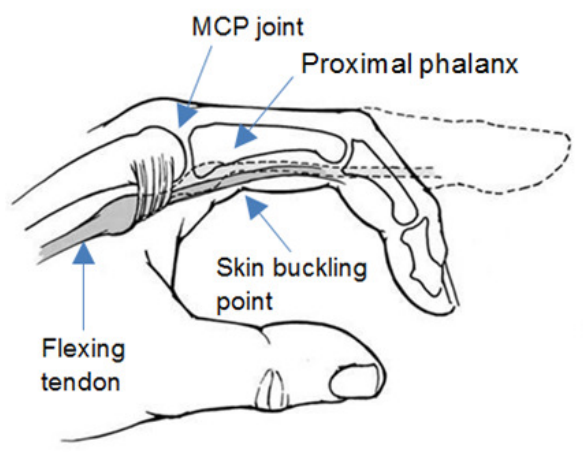

(a)

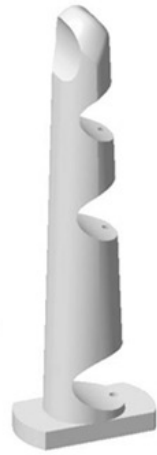

(b)

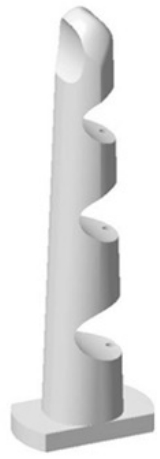

(c)

Fig. 6. (a) Flexion of a human finger, (b) soft finger design with anthropomorphic joint locations and (c) soft finger design with modified metacarpophalangeal (MCP) joint locations.

We have recently designed a soft robotic device made of electroactive polymers which provide not only actuation but also a mechanical structure and a motion conversion mechanism for the device [29]. Although the actuators made of smart materials such as electroactive polymers have a high ratio of force output to actuator weight, their actual force outputs are quite low when used to articulate a functional macro-sized device such as a prosthetic hand. The actuation concepts based on smart materials are more suitable to micro- and mili-domain robotic applications. For this reason, 
we have chosen the tendon cable driven actuation method for the soft monolithic finger. Also, it is suitable for FDM 3D printing method where light weight and durable structures can be fabricated monolithically and all the fabrication steps such as in conventional fabrication methods are eliminated. This actuation method allows underactuation and is easy to implement and sophisticated channel design is not necessary, compared to other possible actuation methods used in recent soft robotic applications which are powered by pressurized air or fluid. While a fluid powered design have advantages over tendon cable actuation such as suitability for modular design, pneumatic actuation requires a pumping unit which can be undesirable if robust and compact design is required. Conventional tendon cable driven robotic fingers and/or hands require a pulley system to pass the tendon cable through, which is not only complicated to assemble but also hard to regularly maintain. Recent studies show that under actuated finger mechanism with tendon cable routing can be fabricated using casting techniques such as the SDM. However, our 3D printed and fully compliant one-piece soft finger eliminates such a complex and multi-component assembly due to its compliant design and monolithic topology.

One must note that the proximal phalanx of a human index finger is longer than the other phalanges. Taking the exact geometric parameters of the human index finger into account while designing an underactuated robotic finger can result in an inefficient grasping. On the other hand, if grasping is one of the design objectives, shorter links (phalanges) of the underactuated (soft) robotic finger can provide more effective and adaptive grasping. Gripping and grasping quantification is a large robotics research area which has been widely studied in the literature dealing with robotic hands with rigid links. Even though grasping efficacy is beyond the scope of this paper, it can simply be assessed by comparing the curvatures of two finger designs: one with anthropomorphic dimensions and the other one with shorter proximal phalanx at their bent (flexion) configurations. While larger objects may have contact with all phalanges of the robotic finger with anthropomorphic dimensions, smaller objects cannot be grasped due to the limits of the flexure joints and the longer proximal phalanges. For this reason, we have studied two different flexure hinge locations for the MCP of the soft finger, as shown in Fig. 6.

\section{Experimental Results and Discussion}

To validate the FE models of the flexure hinges and identify the Young's modulus of the printed thermoplastic, a series of experimental tests were conducted for the 3D printed flexure hinges. Two groups (G1 and G2) of 3D printed flexure hinges were tested and quantitatively compared. Digital models of the five most commonly used types of flexure hinges were designed based on the geometric parameters outlined in Fig. 3.

\section{a. The Design and Fabrication Process}

The flexure hinges for the design of the soft robotic finger with anthropomorphic dimensions were designed using Equations 1-4 and the geometric parameters shown in Fig. 3. The flexure hinge thickness $t$ of $3 \mathrm{~mm}$ was selected and this is the same for all flexure hinges used. While $3 \mathrm{~mm}$ thickness is arbitrary selection and flexure hinges can be thinner or thicker, it should be noted that 
the thickness of the flexure hinge should not buckle under loading. $l$ length of non-symmetric circular, symmetric circular and symmetric corner filleted is $17 \mathrm{~mm}$, and non-symmetric and symmetric elliptical is $16 \mathrm{~mm} . w$ width of the all flexure hinges are same, $20 \mathrm{~mm} . c$ length of nonsymmetric circular, symmetric circular and symmetric corner filleted is designed $8.5 \mathrm{~mm}$, and nonsymmetric and symmetric elliptical is $15 \mathrm{~mm}$. The extruder of the 3D printer was modified to enable regulation of the extruding temperature in order to optimize the printing quality of the thermoplastic elastomer filament. The major differences between the 3D printing conditions of the two groups of flexure hinges are the extrusion temperature and the density of the internal supporting material which determines the porosity in a 3D printed flexure hinge. The two flexure hinges under different 3D printing conditions are shown in Fig. 7. The higher the extrusion temperature, the lower the viscosity of the extruded thermoplastic elastomer. This means that the thickness of the printing patterns enlarges at higher extrusion temperatures and the porosity of the printed part reduces. Flexure hinges were $3 \mathrm{D}$ printed in a room at a standard room temperature $24^{\circ} \mathrm{C}$. The printing stage was preheated at $90{ }^{\circ} \mathrm{C}$, however, the TPU material does not exhibit warping due to thermal stress and stage heating is not compulsory. Nevertheless, a heated stage helps removal of the flexure hinges as it keeps the flexure hinge hotter and softer near the stage surface. Humidity of the room was not measured and it was not controlled as it may not have effect on the properties of the TPU structures 3D printed unless extreme humidity differences happen during printing.

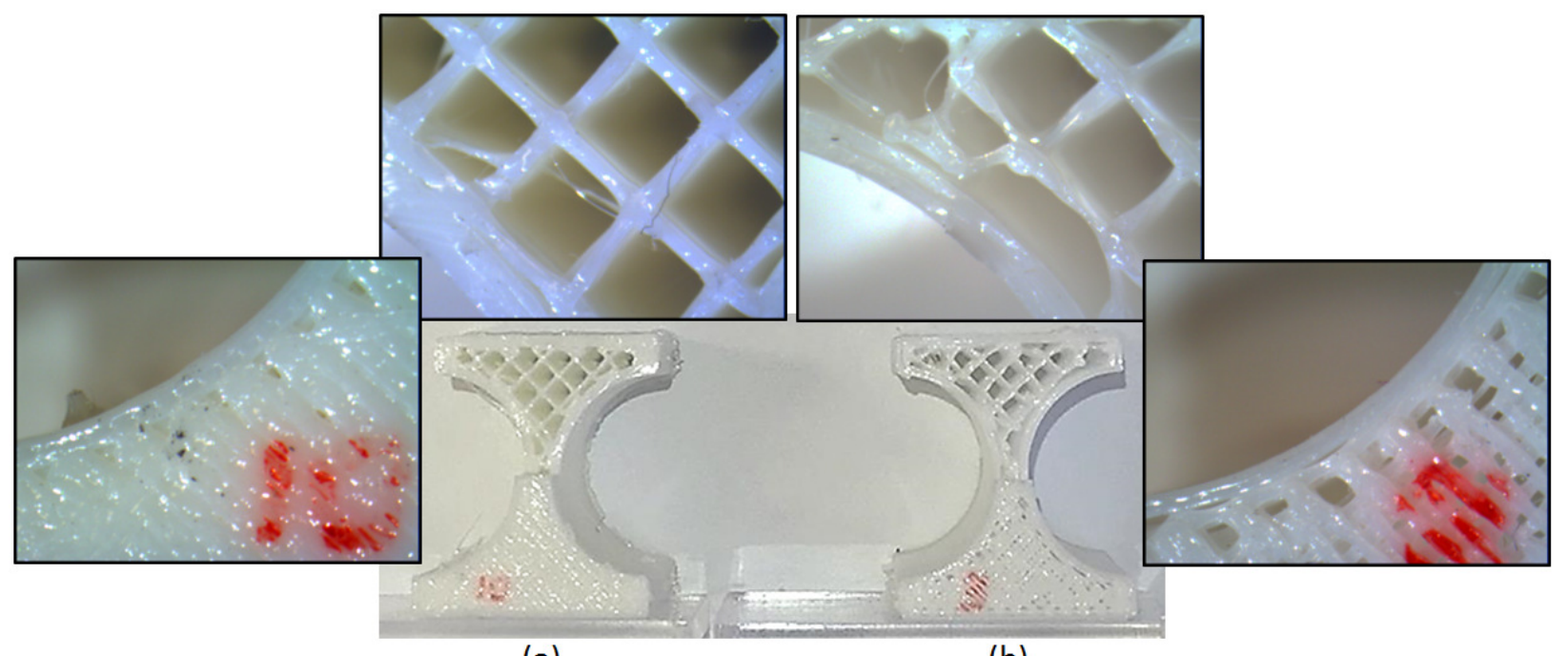

(a)

(b)

Fig. 7. Internal structure of $3 \mathrm{D}$ printed flexure hinges: (a) flexure hinge from the $\mathrm{G} 2$ and (b) from the G1). G2 hinges have more suporting material (printing thickness is larger), and less porosity. The top half of the hinges is sectioned, in parallel to the page plane, to show their internal structure. The top photos show internal morphology and wall thickness, and side photos show surface morphology (magnified photos are taken under the same magnification).

The flexure hinges were tested using a testing platform which designed to regulate the pulling force applied. Another experimental setup was constructed to measure the bending displacement of 
the soft finger with the identified flexure hinge. The experimental setups for displacement and bending measurement are shown in Fig. 8. The flexure hinges are rotated to adjust the pulling force. With a grid paper in the background, each test was captured and the bending displacements of each flexure hinge were then analyzed using the captured images. A set of experimental images of flexure hinges at a bent state is shown in Fig. 9. A similar approach was followed to measure the displacements of the soft finger. The soft finger was fixed on a stage and the tendon cable, which was fixed to the tip of the soft finger and passes through channels in the finger, was attached to a force sensor that is fixed on a micrometer positioning stage. A servo motor is attached the micrometer to control the stage position and therefore, also, the displacement of the tendon cable. By image processing, the recorded videos of the motion of the soft finger from the relaxed to the fully bent state, tip and joint positions (kinematic configurations) were obtained corresponding to the tendon force.

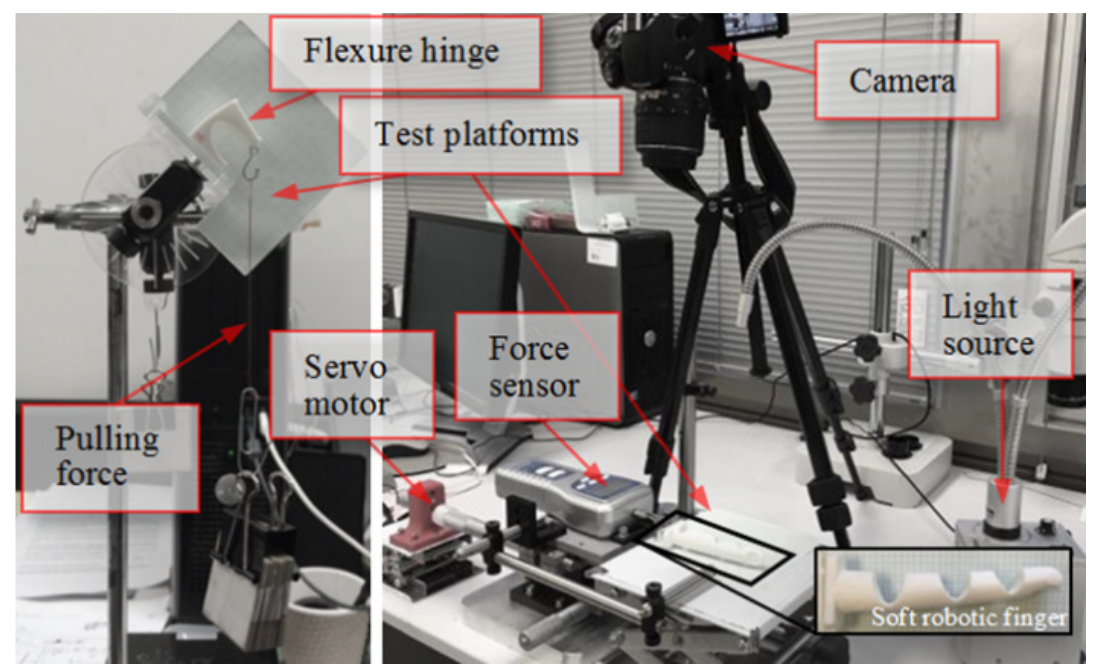

Fig. 8. Experimental setups for the flexure hinge (on the left) and the soft robotic finger (on the right) for the displacement and bending angle measurements.

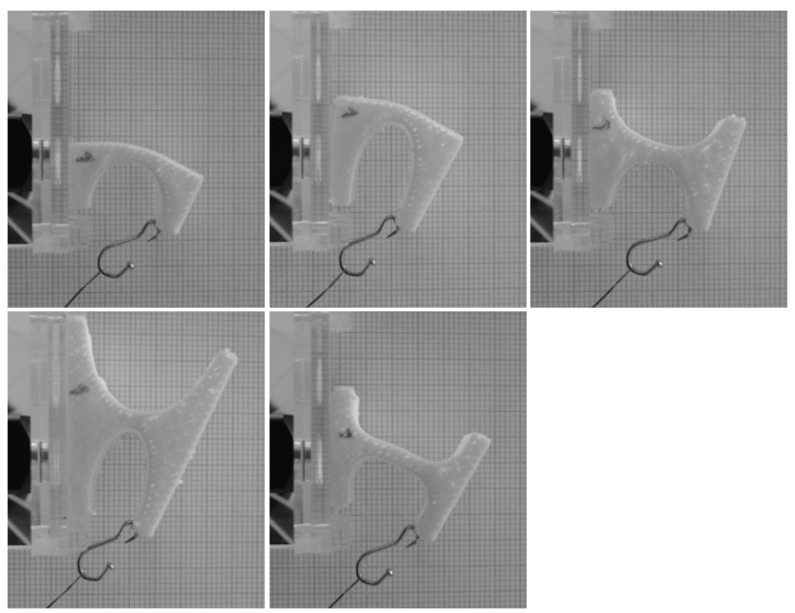

Fig. 9. Experimental images of the flexure hinges at their bent states under same force input. 


\section{b. Experimental Validation}

We conducted a series of experiments to estimate the effective Young's modulus of the group of flexure hinges printed under the same conditions. The effective Young's moduli of the thermoplastic elastomer material for both groups are estimated using optimization toolbox in ANSYS where the Young's modulus is parametrized against experimental displacements of the flexure hinges. By numerically comparing the experimental and simulation results, the effective Young's moduli of the thermoplastic elastomer material for the G1 and G2 flexure hinges are estimated as $3.74 \mathrm{MPa}$ and $11 \mathrm{MPa}$, respectively. It should be noted that, as the flexure hinges are not homogeneous and very sensitive to printing conditions, the estimated Young's modulus is valid only for the flexure hinges fabricated under the same printing conditions. To test the bending and displacement capabilities of a flexure hinge, the flexure hinge was fixed to the testing platform and rotated $45^{\circ}$ counter clockwise. A number of dead weights were hung on it using a hook to the flexure hinge's internal edge of the flexure groove as illustrated in Fig. 8 (on the left). This configuration provides the pulling force with $45^{\circ}$ with the longitudinal axis of the flexure hinge. The initial and the final states of the flexure hinge were captured and extracted from the images by analyzing them in order to obtain the bending angle and the displacement of the flexure hinge under each pulling force. The bending angle and the displacement results with a linear regression and shaded error bars (i.e. standard deviation) of each test group of the flexure hinges are shown in Figs. 10 and 11, respectively. The average stiffness of each flexure hinge corresponding to the data in Figs. 10 and 11 is presented in Table 1. These results show that the stiffness of the non-symmetric elliptical hinge is the smallest in both groups of hinges. In other words, the non-symmetric elliptical hinge shows the largest angular and rectilinear displacement under the same force input. FE models are designed with the same loading conditions. Optimization toolbox in ANSYS is used to estimate the effective Young's modulus of the flexure hinges using the experimentally obtained displacement results.
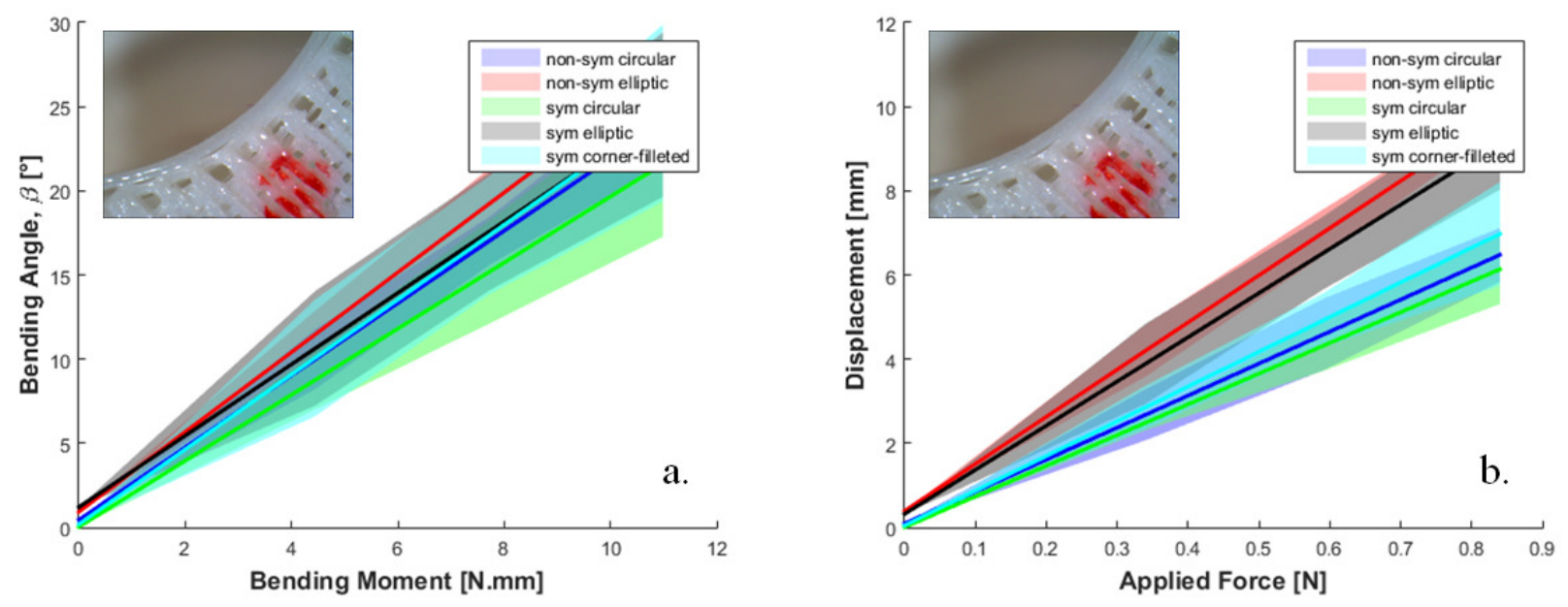
Fig. 10. a. Bending angle and b. displacement results (average and standard deviations) of the G1 flexure hinges (inlet photos show surface of the G1 flexure hinge under microscope).
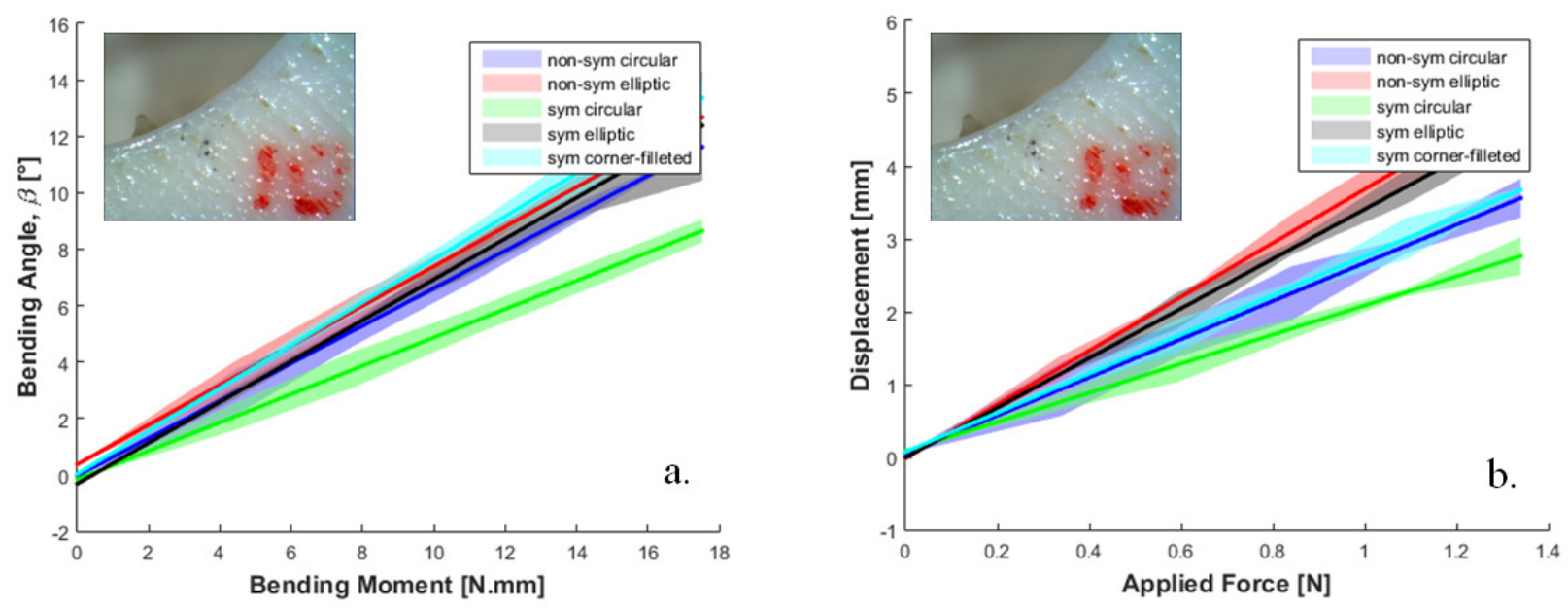

Fig. 11. a. Bending angle and b. displacement results (average and standard deviations) of the G2 flexure hinges (inlet photos show surface of the G2 flexure hinge under microscope).

Table 1. Stiffness of the 3D printed flexure hinges

\begin{tabular}{|c|c|c|c|c|c|}
\hline & \multicolumn{2}{|c|}{ G1 } & \multicolumn{2}{|c|}{ G2 } \\
\hline & & Fig.10a & Fig.10b & Fig.11a & Fig.11b \\
\hline & & $\mathrm{Nmm} / \mathrm{deg}$ & $\mathrm{N} / \mathrm{mm}$ & $\mathrm{Nmm} / \mathrm{deg}$ & $\mathrm{N} / \mathrm{mm}$ \\
\hline \multirow{2}{*}{$\begin{array}{l}\dot{1} \\
Z \\
Z\end{array}$} & Circular & 0.467 & 0.132 & 1.510 & 0.381 \\
\hline & Elliptical & 0.423 & 0.089 & 1.430 & 0.270 \\
\hline \multirow{3}{*}{ 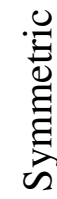 } & Circular & 0.511 & 0.137 & 1.997 & 0.500 \\
\hline & Elliptical & 0.473 & 0.095 & 1.386 & 0.293 \\
\hline & Corner-filleted & 0.451 & 0.121 & 1.320 & 0.372 \\
\hline
\end{tabular}

To further validate the FE models and moduli of the elasticity identified for G1 and G2 hinges, we compared the bending outputs (the rectilinear displacement along the direction of the pulling force, and bending angle) of the non-symmetric circular hinges and non-symmetric elliptical hinges. The numerical and experimental results for G1 and G2 hinges are shown in Figs. 12 and 13, respectively. With reference to these results, there is a better correlation between the predicted displacement results and corresponding experimental results of the G2 flexure hinges, compared to the G1 hinges. The predicted displacement results are obtained using FE models of the flexure 
hinges. We postulate that this can be due to the fact that the porosities in the G2 are less than those of G1 - providing a more uniform structure, thus the G2 hinges are denser than the G1 hinges. The higher the density of the internal supporting structure, the higher the homogeneity of the 3D printed part, but this will increase the total mass of the flexure hinge, and consequently that of the soft finger. It should be noted that the flexure hinges in the soft robotic finger are articulated with the G2 flexure hinges, which are denser and can potentially reduce such a discrepancy between the experimental and numerical results. Another implication of this discrepancy between the calculated and measured results is that treating the hinge material as fully dense with a fitted Young's modulus does not adequately reflect the more complex porous internal structure. With the aim of this study to identify the flexure hinge morphology with lower stiffness, providing larger displacement for lower applied force, under the same printing conditions, the non-symmetric elliptical flexure hinges are more flexible than other type of 3D printed flexure hinges as the results in Figs. 12 and 13 also suggest. However, the non-symmetric elliptical flexure hinge with printing conditions of the G2 has been chosen for establishing the soft robotic fingers due to the ability to store higher elastic potential energy (i.e., the area under the force versus displacement curves) than G1 and prevent any potential buckling in the finger under loading. It is also supported by the results in Figs. 10 and 11.
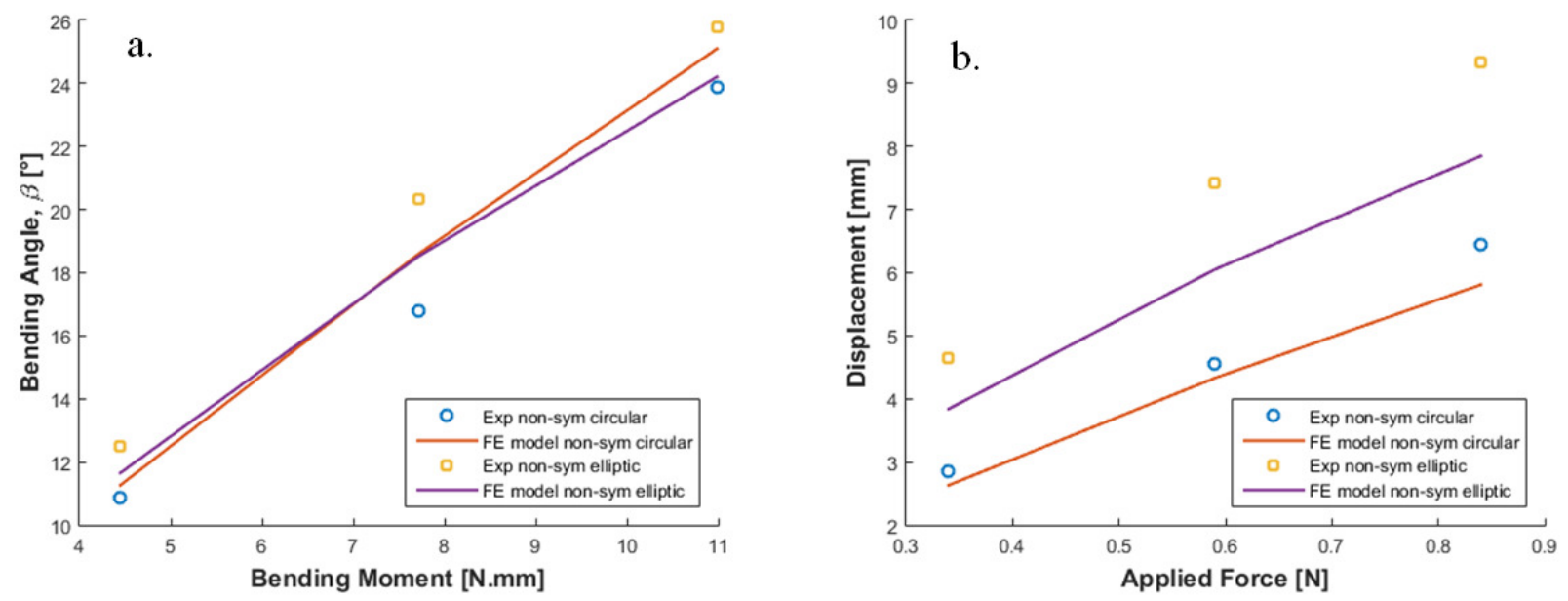

Fig. 12. Experimental and numerical bending outputs of the non-symmetric circular hinges and non-symmetric elliptical hinges in the $\mathrm{G} 1$; $a$. bending angle and $\mathrm{b}$. displacement (corresponding to the rectilinear deflection from the configuration A to the configuration A' in Fig.4). 

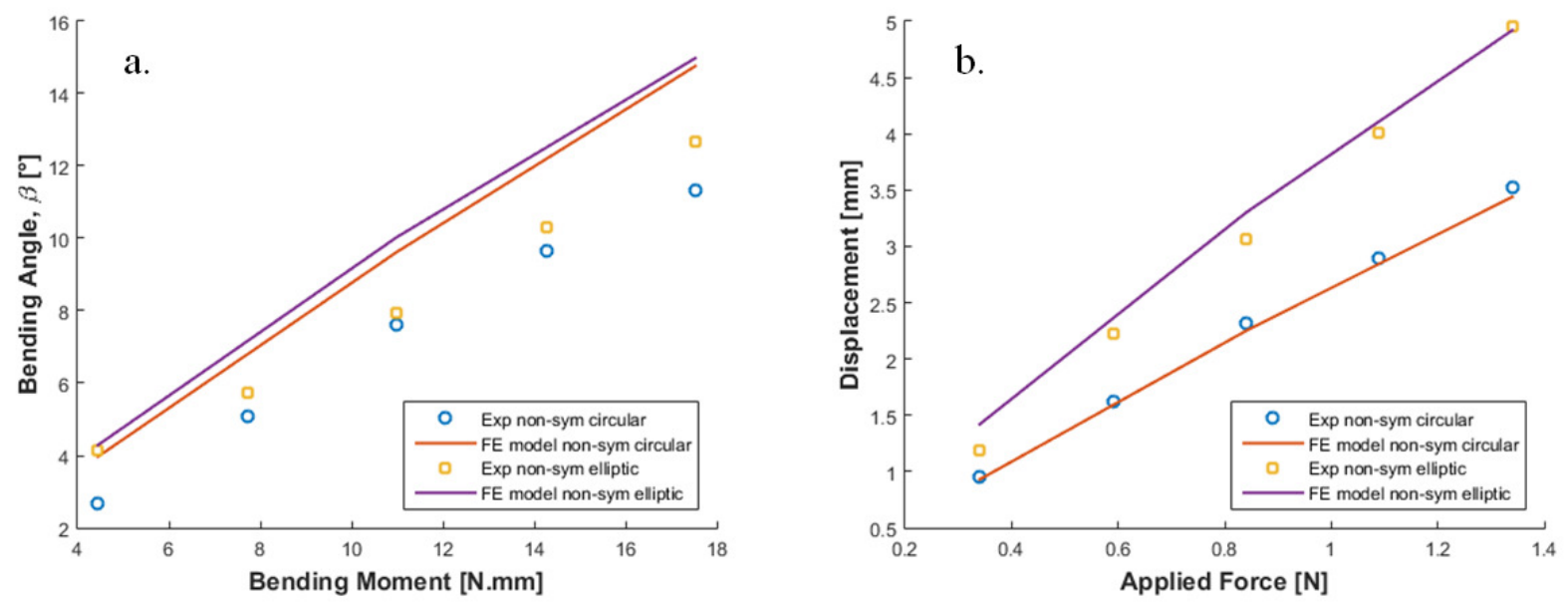

Fig. 13. Experimental and numerical bending outputs of the non-symmetric circular hinges and non-symmetric elliptical hinges in the $\mathrm{G} 2$; a. bending angle and b. displacement (corresponding to the rectilinear deflection from the configuration A to the configuration A' in Fig.4).

\section{Potential Applications: A Soft Robotic Gripper with Fully Compliant and Adaptive Fingers}

We created a soft robotic finger using the non-symmetric elliptical flexure hinges. With the geometric parameters listed in Fig.5, the soft robotic finger was fabricated under the same printing conditions of flexure hinges in the G2. We have developed an image processing algorithm to determine the bending displacements of the soft finger. The specified data points, i.e. the center positions of the flexure hinges and the tip of the finger, were extracted from the recorded videos of the finger. As stated above, the geometric parameters of a human index finger have been used as a reference to design the soft robotic finger. Designs of a robotic finger having the proximal phalanx (link) of the finger longer than the distal phalanges reduces effectiveness of power grasping prehensions (i.e. cylindrical grasp, spherical grasp) of the robotic finger in a prosthetic hand [36]. This is eliminated in the real human index finger by the skin, buckling in shorter distances, resulting in a more conformal grasping. According to Kargov et al. [36], adaptive prosthetic hands provide a larger contact area. On the other hand, non-adaptive prosthetic hands have very limited contact with the object grasped in the metacarpal region [36]. This means that when sizing a prosthetic hand or its fingers, the lengths of the metacarpal and the proximal phalanx of the finger should be kept shorter and similar to the lengths of the distal links. For this reason, we have designed a shorter proximal phalanx for the soft monolithic robotic finger to show the difference in grasping. The closer the lengths of phalanges, the higher the conformability of the soft monolithic finger. The sequential images of the flexion of both robotic fingers are presented in Figs. 14 and 15. Although the thickness of the flexure hinges is the same for each flexure hinge of the soft robotic finger, flexion rates are different due to the acting bending moment difference at the flexure hinges. There are two main reasons for this difference. First, the moment arm which is the length between flexure hinge mid-point and tendon cable is different for each flexure hinge. Second, friction in the channels accumulates from the base to the tip of the finger, thus the tendon force decreases towards the tip 
of the finger. By considering these effects on the flexion of the flexure hinges, the soft robotic finger's hinges can be designed based on the desired flexion and extension motion. The joint and tip positions of the fingers retrieved using image processing are provided in Fig. 16, which shows the flexion and extension motion paths of the soft robotic fingers. A hysteresis effect is observed in the flexion-extension motion of the soft fingers which, we believe, is mainly due to the friction between the tendon cable and the soft channel. It must be noted that this effect is more obvious towards the tip of the finger (hysteresis of tip position is the largest in Fig. 16) as friction forces in the channels accumulate. This hysteresis is also more obvious for the soft robotic finger with anthropomorphic dimensions (Fig. 16 orange line with circle marks) because it has larger contact area in its proximal phalanx.
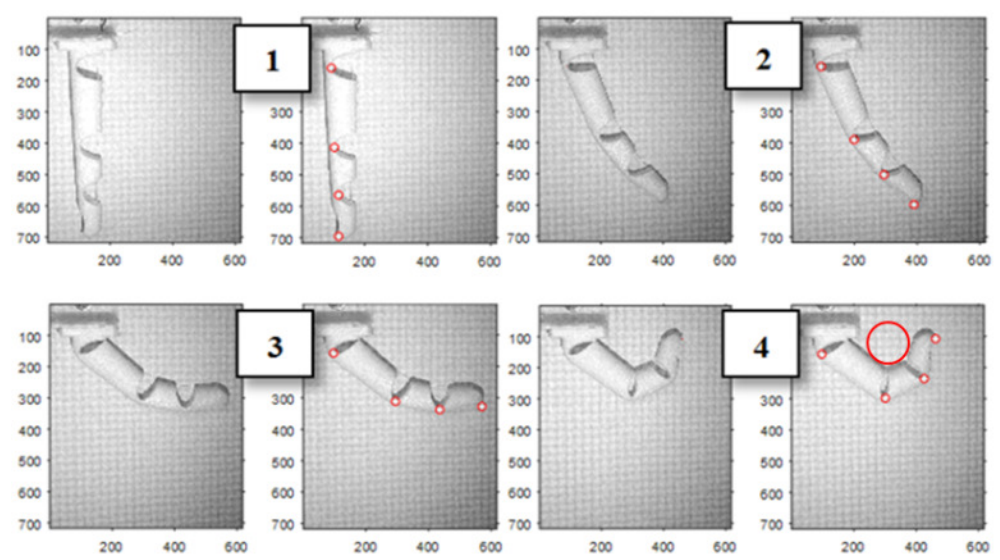

Fig. 14. Sequential bending motion frames of the real robotic finger with the anthropomorphic geometric parameters. An imaginary circular object with $0.18 \mathrm{~mm}^{-1}$ curvature is plotted for grasping comparison.
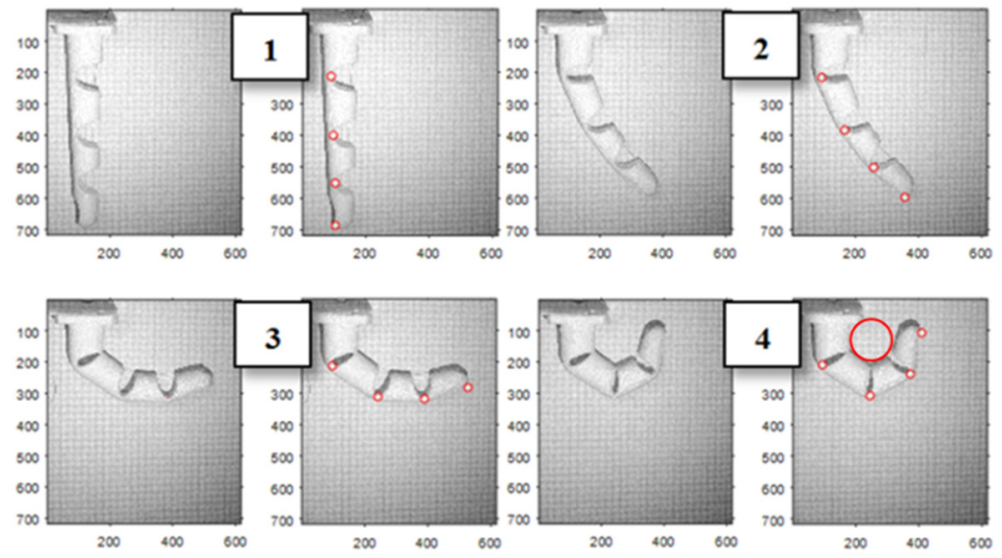

Fig. 15. Sequential bending motion frames of the real robotic finger with the modified MCP joint location. An imaginary circular object with $0.18 \mathrm{~mm}^{-1}$ curvature is plotted for grasping comparison. 


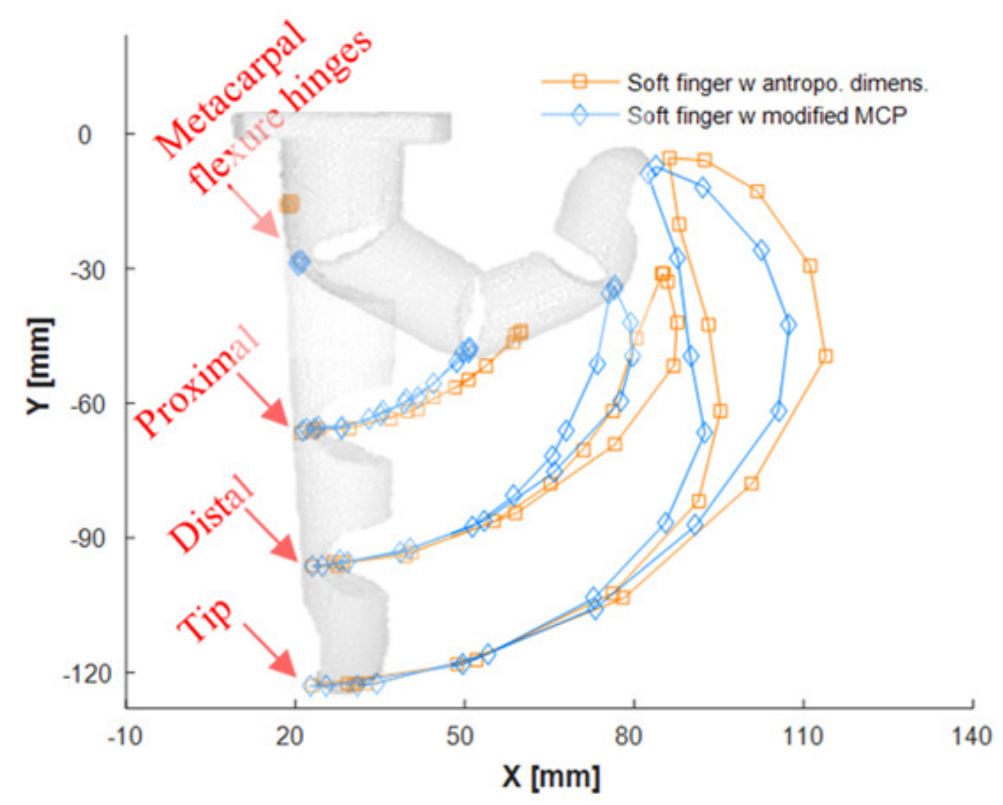

Fig. 16. Experimental displacement results for the joints and tip of the soft robotic fingers with anthropomorphic geometric parameters (orange line with circle marks) and with modified MCP joint location (blue line with diamond marks). The fingers with the modified MCP joint are in the background.

In the FE models of the soft robotic fingers, we have considered the Capstan effect [37], which describes the relation between the input and output tensile forces ( $F_{\text {in }}$, and $F_{\text {out }}$ ) of a flexure hinge, and the friction coefficient $\mu$ between the tendon cable and the soft channels in the finger's structure. $F_{\text {in }}$ and $F_{\text {out }}$ are the tension forces in both ends of the tendon cable passing over the hinge. For the contact angle $\theta$ between the tendon cable and the sliding surface in each flexure, the Capstan effect is given by

$$
F_{\text {out }}=\frac{F_{\text {in }}}{e^{\mu \theta}}
$$

The initial and final angular displacements of the stiffer parts (links) of the soft robotic finger were determined experimentally. These angular displacements are then used to calculate tendon forces, therefore bending moments, for each flexure hinge in the model. The soft material used to 3D print the fingers is new and its mechanical properties, such as friction coefficient, to the best of our knowledge, are not available. We therefore experimentally identified the friction coefficient between the tendon cable and the soft finger channels which is to be 0.1 , by comparing the experimental bending displacements obtained from the finger and the FE model. The FE models of the soft fingers are constructed without an actual tendon cable; instead equivalent bending moments are calculated for each joint (flexure hinge) of the fingers. A comparison of the soft robotic fingers with anthropomorphic geometric parameters and with modified MCP hinge location and corresponding FE models are presented in Fig. 17 for the fully flexed state of the fingers. The friction is quite high between the soft channels and the tendon cable, especially towards the fully flexed state of the finger. Even though the proximal and middle phalanges of the FE models show 
quite good correspondence with the corresponding experimental bending results, the distal phalanx still generates an acceptable correlation between the numerical and experimental bending results. This might be due to the tendon cable forces, therefore bending moments, calculated using the Capstan friction effect. As also these results suggest, the magnitude of the bending moment in the distal interphalangeal joint of the FE model is less than that of the bending moment in the real soft finger due to the accumulated sum of the friction forces reflected on this distal joint. Advanced analyses will be done in the future work to experimentally obtain frictional forces between tendon cable and the channels and calculate bending moments of the hinges more precisely.
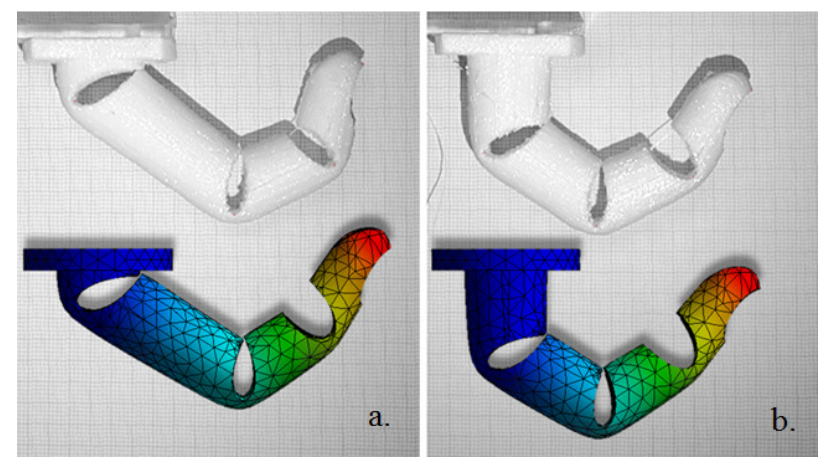

Fig. 17. Comparison of the experimental and FE models of the soft monolithic robotic fingers with a. anthropomorphic geometric parameters and b. with modified MCP joint location.

In order to demonstrate conformability of proposed soft monolithic fingers, we constructed a 3finger soft robotic gripper which can softly handle objects with irregular shapes, as shown in Fig. 18. Having in mind that the shorter link lengths provide more conformal grasping and lower friction in the tendon channels, two of the fingers were designed with four flexure hinges and third finger with same dimensions as the soft finger with modified MCP joint location. This gripper is activated by one actuator only, demonstrating the validity of the underactuation concept. Further analyses and performance quantification of the soft robotic gripper is left for future studies.
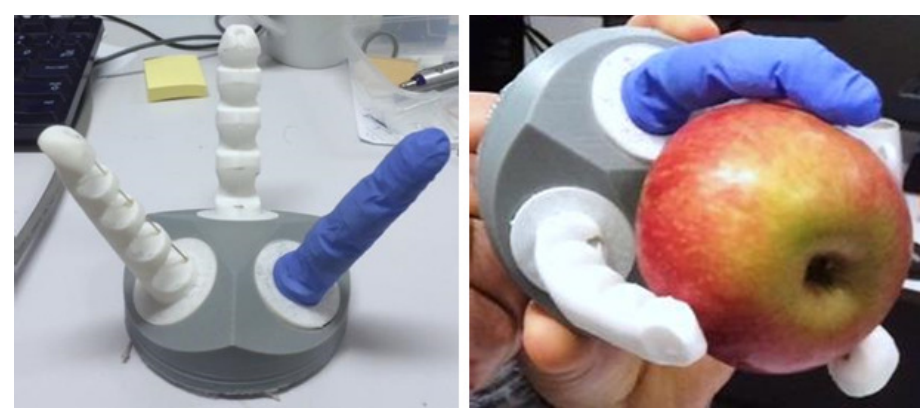

Fig. 18. Fully compliant and adaptive 3 -finger soft robotic gripper.

\section{Conclusion and Future Work}

A number of commonly used single-axis flexure hinges have been investigated in terms of their angular and rectilinear displacements. The flexure hinges were fabricated using FDM-based 
additive manufacturing. FE models of the flexure hinges were developed for two purposes. First, the Young's modulus for the printed thermoplastic elastomer was estimated by comparing the FE results with the experimental displacement results. Next, the Young's modulus was used to estimate the bending behaviour of the soft monolithic robotic finger which had been fabricated under the same 3D printing conditions as the flexure hinge type identified. The non-symmetric elliptical flexure hinges output a larger mechanical work under the same loading conditions compared to the other types of flexure hinges. Using non-symmetric elliptical flexure hinges, we developed and fabricated two soft and one-piece robotic fingers: one with exact anthropomorphic geometric parameters and the other with a modified MCP hinge location (with shorter proximal phalanx). The closer the lengths of phalanges, the higher the conformability of the soft monolithic finger. The displacement results of the flexure hinges and the fingers were obtained using an image processing system. We also proposed a potential application for the soft monolithic fingers as a soft robotic gripper because it has a great potential to handle both very fragile objects as well as objects with unknown shapes which requires a lot of sensing feedback and precise position control if a conventional rigid robotic gripper is employed. Such a soft and monolithic robotic gripper is easy to build and requires less control due to the adaptability of the fingers to the object grasped. The soft fingers show a hysteresis in their flexion-extension motion, which is likely due to the viscoelastic properties of the thermoplastic material and more possibly the friction force of the tendon cables in the sliding channels.

In future work, we will investigate and attempt to reduce the hysteresis in the soft robotic finger. We also plan to integrate a soft sensing capability into the soft robotic finger so that its bending behaviour can be determined without using an external displacement measurement system such as the image processing system and its force output would be able to be estimated and controlled through the sensing system.

\section{Acknowledgments}

This work has been supported by ARC Centre of Excellence for Electromaterials Science (Grant No. CE140100012), the University of Wollongong and the Australian National Fabrication Facility (ANFF) Materials Node. The authors wish to gratefully acknowledge the help of Dr. Madeleine Strong Cincotta in the final language editing of this paper.

\section{Author Disclosure}

The authors declare no competing financial interests.

Correspondence regarding this manuscript should be directed to:

Professor Gursel Alici 
School of Mechanical, Materials and Mechatronic Engineering, and Intelligent Polymer Research Institute, ARC Centre of Excellence for Electromaterials Science, University of Wollongong, AIIM Facility, NSW, 2522, Australia.

gursel@uow.edu.au

\section{References}

[1] N. Lobontiu, Compliant Mechanisms: Design of Flexure Hinges: CRC Press, 2002.

[2] N. D. Mankame and G. K. Ananthasuresh, "A Novel Compliant Mechanism for Converting Reciprocating Translation Into Enclosing Curved Paths," Journal of Mechanical Design, vol. 126, pp. 667-672, 2004.

[3] J. Speich and M. Goldfarb, "A compliant-mechanism-based three degree-of-freedom manipulator for small-scale manipulation," Robotica, vol. 18, pp. 95-104, 2000.

[4] Y.-M. Moon, "Bio-mimetic design of finger mechanism with contact aided compliant mechanism," Mechanism and Machine Theory, vol. 42, pp. 600-611, 2007.

[5] L. Rubbert, S. Caro, J. Gangloff, and P. Renaud, "Using Singularities of Parallel Manipulators to Enhance the Rigid-Body Replacement Design Method of Compliant Mechanisms," Journal of Mechanical Design, vol. 136, p. 051010 (9pp), 2014.

[6] M. L. Culpepper, C. M. DiBiasio, R. M. Panas, S. Magleby, and L. L. Howell, "Simulation of a carbon nanotube-based compliant parallel-guiding mechanism: A nanomechanical building block," Applied Physics Letters, vol. 89, p. 203111 (6pp), 2006.

[7] P. S. Sreetharan, J. P. Whitney, M. D. Strauss, and R. J. Wood, "Monolithic fabrication of millimeter-scale machines," Journal of Micromechanics and Microengineering, vol. 22, p. 055027 (6pp), 2012.

[8] S. Hirose and M. Mori, "Biologically Inspired Snake-like Robots," in Robotics and Biomimetics, 2004. ROBIO 2004. IEEE International Conference on, 2004, pp. 1-7.

[9] B. Atakan, A. M. Erkmen, and I. Erkmen, "3-D grasping during serpentine motion with a snake-like robot," in Proceedings of the Sixth IASTED International Conference on Robotics and Applications, 2005, pp. 46-51.

[10] C. D. Onal and D. Rus, "A modular approach to soft robots," in Biomedical Robotics and Biomechatronics (BioRob), 2012 4th IEEE RAS \& EMBS International Conference on, 2012, pp. 1038-1045.

[11] R. Kang, A. Kazakidi, E. Guglielmino, D. T. Branson, D. P. Tsakiris, J. A. Ekaterinaris, et al., "Dynamic model of a hyper-redundant, octopus-like manipulator for underwater applications," in Intelligent Robots and Systems (IROS), 2011 IEEE/RSJ International Conference on, 2011, pp. 4054-4059.

[12] C. Laschi, B. Mazzolai, V. Mattoli, M. Cianchetti, and P. Dario, "Design of a biomimetic robotic octopus arm," Bioinspiration \& Biomimetics, vol. 4, p. 015006 (8pp), 2009.

[13] Y. Yekutieli, R. Sagiv-Zohar, R. Aharonov, Y. Engel, B. Hochner, and T. Flash, "Dynamic Model of the Octopus Arm. I. Biomechanics of the Octopus Reaching Movement," Journal of Neurophysiology, vol. 94, pp. 1443-1458, 2005.

[14] M. W. Hannan and I. D. Walker, "Kinematics and the Implementation of an Elephant's Trunk Manipulator and Other Continuum Style Robots," Journal of Robotic Systems, vol. 20, pp. 45-63, 2003. 
[15] O. Salomon and A. Wolf, "Inclined Links Hyper-Redundant Elephant Trunk-Like Robot," Journal of Mechanisms and Robotics-Transactions of the ASME, vol. 4, p. 045001 (6pp), 2012.

[16] S. Sangok, C. D. Onal, C. Kyu-Jin, R. J. Wood, D. Rus, and K. Sangbae, "Meshworm: A Peristaltic Soft Robot With Antagonistic Nickel Titanium Coil Actuators," Mechatronics, IEEE/ASME Transactions on, vol. 18, pp. 1485-1497, 2013.

[17] S. M. Felton, M. T. Tolley, C. D. Onal, D. Rus, and R. J. Wood, "Robot Self-Assembly by Folding: A Printed Inchworm Robot," in 2013 IEEE International Conference on Robotics and Automation (ICRA), 2013, pp. 277-282.

[18] L. Huai-Ti, G. L. Gary, and T. Barry, "GoQBot: a caterpillar-inspired soft-bodied rolling robot," Bioinspiration \& Biomimetics, vol. 6, p. 026007 (14pp), 2011.

[19] M. T. Tolley, R. F. Shepherd, B. Mosadegh, K. C. Galloway, M. Wehner, M. Karpelson, et al., "A Resilient, Untethered Soft Robot," Soft Robotics, vol. 1, pp. 213-223, 2014.

[20] J. M. Germann, A. Maesani, R. Pericet Camara, and D. Floreano, "Soft Cells for Programmable Self-Assembly of Robotic Modules," Soft Robotics, vol. 1, pp. 239-245, 2014.

[21] B. Mosadegh, P. Polygerinos, C. Keplinger, S. Wennstedt, R. F. Shepherd, U. Gupta, et al., "Pneumatic Networks for Soft Robotics that Actuate Rapidly," Advanced Functional Materials, vol. 24, pp. 2163-2170, 2014.

[22] A. D. Marchese, C. D. Onal, and D. Rus, "Autonomous Soft Robotic Fish Capable of Escape Maneuvers Using Fluidic Elastomer Actuators," Soft Robotics, vol. 1, pp. 75-87, 2014.

[23] K. C. Galloway, K. P. Becker, B. Phillips, J. Kirby, S. Licht, D. Tchernov, et al., "Soft Robotic Grippers for Biological Sampling on Deep Reefs," Soft Robotics, vol. 3, pp. 2333, 2016.

[24] A. M. Dollar and R. D. Howe, "A robust compliant grasper via shape deposition manufacturing," IEEE/ASME Transactions on Mechatronics, vol. 11, pp. 154-161, 2006.

[25] J. Gafford, Y. Ding, A. Harris, T. McKenna, P. Polygerinos, D. Holland, et al., "Shape Deposition Manufacturing of a Soft, Atraumatic, Deployable Surgical Grasper1," Journal of Medical Devices, vol. 8, p. 030927 (3pp), 2014.

[26] Available: http://sssa.bioroboticsinstitute.it/research/softrobotics

[27] Available: http://softroboticsinc.com/

[28] Available: http://www.festo.com/cms/en_corp/13655.htm

[29] R. Mutlu, G. Alici, and W. Li, "A Soft Mechatronic Micro-Stage Mechanism Based on Electroactive Polymer Actuators," IEEE/ASME Transactions on Mechatronics, vol. 21, pp. 1467-1478, 2016.

[30] R. Mutlu, G. Alici, and W. Li, "Three-Dimensional Kinematic Modeling of Helix-Forming Lamina-Emergent Soft Smart Actuators Based on Electroactive Polymers," IEEE Transactions on Systems, Man, and Cybernetics: Systems, pp. 1-12, doi: 10.1109/TSMC.2016.2523940, 2016.

[31] S. Tibbits, "4D Printing: Multi-Material Shape Change," Architectural Design, vol. 84, pp. 116-121, 2014.

[32] S. E. Bakarich, R. Gorkin, M. i. h. Panhuis, and G. M. Spinks, "4D Printing with Mechanically Robust, Thermally Actuating Hydrogels," Macromolecular Rapid Communications, vol. 36, pp. 1211-1217, 2015. 
[33] R. G. E. Clement, K. E. Bugler, and C. W. Oliver, "Bionic prosthetic hands: A review of present technology and future aspirations," The Surgeon, vol. 9, pp. 336-340, 2011.

[34] R. Mutlu, G. Alici, M. in het Panhuis, and G. Spinks, "Effect of flexure hinge type on a 3D printed fully compliant prosthetic finger," in Advanced Intelligent Mechatronics (AIM), 2015 IEEE International Conference on, 2015, pp. 790-795.

[35] H. W. Kay and M. Rakic, "Specifications for Electromechanical Hands," in Proceedings of the 4th International Symposium on the External Control of Human Extremities, 1972, pp. 137-155.

[36] A. Kargov, C. Pylatiuk, J. Martin, S. Schulz, and L. Döderlein, "A comparison of the grip force distribution in natural hands and in prosthetic hands," Disability and Rehabilitation, vol. 26, pp. 705-711, 2004.

[37] A. K. Srivastava, Engineering Mechanics. Lucknow, IND: Word-Press, 2009. 